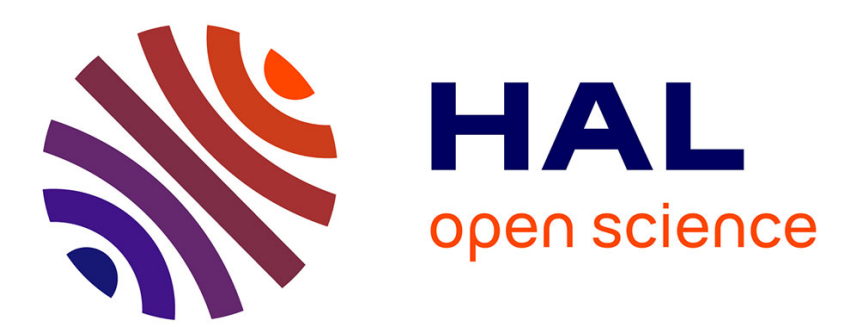

\title{
Hybrid QM/MM Molecular Dynamics with AMOEBA Polarizable Embedding
}

\author{
Daniele Loco, Louis Lagardère, Stefano Caprasecca, Filippo Lipparini, \\ Benedetta Mennucci, Jean-Philip Piquemal
}

\section{> To cite this version:}

Daniele Loco, Louis Lagardère, Stefano Caprasecca, Filippo Lipparini, Benedetta Mennucci, et al.. Hybrid QM/MM Molecular Dynamics with AMOEBA Polarizable Embedding. Journal of Chemical Theory and Computation, 2017, 10.1021/acs.jctc.7b00572 . hal-01571619v2

HAL Id: hal-01571619

https://hal.science/hal-01571619v2

Submitted on 25 Aug 2017

HAL is a multi-disciplinary open access archive for the deposit and dissemination of scientific research documents, whether they are published or not. The documents may come from teaching and research institutions in France or abroad, or from public or private research centers.
L'archive ouverte pluridisciplinaire HAL, est destinée au dépôt et à la diffusion de documents scientifiques de niveau recherche, publiés ou non, émanant des établissements d'enseignement et de recherche français ou étrangers, des laboratoires publics ou privés. 


\title{
Hybrid QM/MM Molecular Dynamics with AMOEBA Polarizable Embedding
}

\author{
Daniele Loco, ${ }^{\dagger}$ Louis Lagardère, ${ }^{\dagger}$ Stefano Caprasecca, ${ }^{\dagger}$ Filippo Lipparini, ${ }^{*}, \S, I \odot$ Benedetta Mennucci, ${ }^{*}, \dagger \odot$ \\ and Jean-Philip Piquemal ${ }^{*}, \|, \perp, \#\left({ }_{0}\right.$
}

${ }^{\dagger}$ Dipartimento di Chimica e Chimica Industriale, Università di Pisa, via G. Moruzzi 13, I-56124 Pisa, Italy

${ }^{\ddagger}$ UPMC Univ. Paris 06, Institut des Sciences du Calcul et des Données, F-75005, Paris, France

${ }^{\S}$ Institut für Physikalische Chemie, Universität Mainz, Duesbergweg 10-14, D-55128 Mainz, Germany

"UPMC Univ. Paris 06, UMR7616, Laboratoire de Chimie Théorique, F-75005, Paris, France

${ }^{\perp}$ Institut Universitaire de France, Paris Cedex 05, 75231, France

${ }^{\#}$ Department of Biomedical Engineering, The University of Texas at Austin, Austin, Texas 78712, United States

\section{Supporting Information}

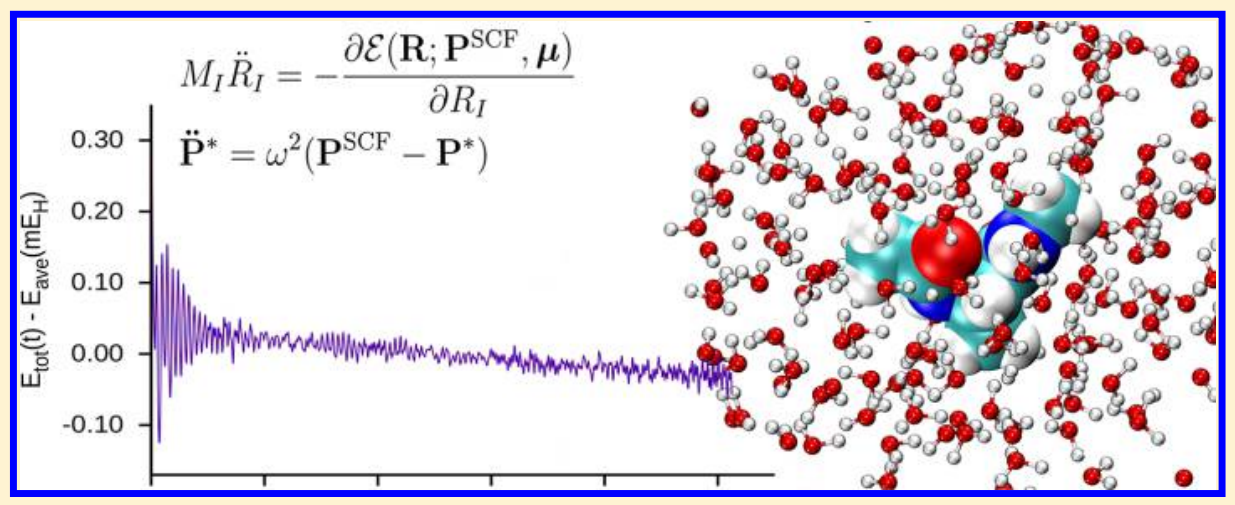

ABSTRACT: We present the implementation of a Born-Oppenheimer (BO) hybrid quantum mechanics/molecular mechanics (QM/MM) molecular dynamics (MD) strategy using density functional theory (DFT) and the polarizable AMOEBA force field. This approach couples the Gaussian and Tinker suite of programs through a variational formalism allowing for a full selfconsistent relaxation of both the AMOEBA induced dipoles and the DFT electron density at each MD step. As the DFT SCF cycles are the limiting factor in terms of computational efforts and $\mathrm{MD}$ stability, we focus on the latter aspect and compare the time-reversible $\mathrm{BO}$ (TR-BO) and the extended BO Lagrangian approaches (XL-BO) to the MD propagation. The XL-BO approach allows for stable, energy-conserving trajectories offering various perspectives for hybrid simulations using polarizable force fields.

\section{INTRODUCTION}

In recent years many efforts have been devoted to improve both the efficiency and the applicability of polarizable molecular mechanics (MM) force fields (FF). ${ }^{1-3}$ In contrast to standard force fields, polarizable ones include many-body effects, which makes them in principle more flexible and accurate. Naturally, a fully classical description, even if including polarization, is not sufficient for many important chemical and physical problems, such as the study of chemical reactivity and photoinduced processes. In that context, a hybrid $\mathrm{QM} / \mathrm{MM}$ approach that couples a polarizable FF with a quantum mechanical (QM) approach represents a very promising strategy as it combines the computational efficiency of an accurate classical model with the required quantum description of the subsystem of interest. Many examples in this direction have been presented so far in the literature, where the polarizable FF can be obtained in terms of fluctuating charges, ${ }^{4-8}$ drude oscillators, ${ }^{9,10}$ or induced dipoles. ${ }^{11-19}$ In the framework of induced dipoles formulations, we recently presented a polarizable $\mathrm{QM} / \mathrm{MM}$ implementation based on density functional theory (DFT) and the AMOEBA polarizable FF. ${ }^{20}$ Such an implementation is based on a variational formalism ${ }^{21,22}$ and couples the induced dipoles and the electron density in a fully self-consistent way. Both groundand excited-state energies have been presented; the latter are obtained within the framework of time-dependent DFT (TD$\mathrm{DFT}$ ), either in a linear response or in a state-specific picture. Our work complements several other QM/AMOEBA implementations within various other suite of programs, such as LICHEM, ${ }^{23}$ ONETEP/TINKER ${ }^{24}$ and the Q-Chem/ LibEFP interface. ${ }^{25}$

Received: June 4, 2017

Published: July 31, 2017 
In our last work, however, the QM/AMOEBA computations were performed on snapshots obtained from a purely classical molecular dynamics (MD) simulation. In other words, the MD and the $\mathrm{QM} / \mathrm{MM}$ calculations were decoupled and performed using different methods. We pursue here a genuine $\mathrm{QM} / \mathrm{MM}$ MD strategy, which is achieved by coupling together the Tinker $^{26}$ MD package and the Gaussian ${ }^{27}$ suite of programs and by implementing analytical gradients for the polarizable QM/AMOEBA energy.

QM/MM MD simulations ${ }^{28-31}$ have been proposed within both Born-Oppenheimer MD (BOMD) ${ }^{32}$ and Lagrangian Car-Parrinello $\mathrm{MD}$ (CPMD). ${ }^{33}$ In BOMD the electronic $\mathrm{QM}(/ \mathrm{MM})$ equations are solved at each time step to obtain the ground-state potential energy and forces acting on the nuclei. In CPMD the electronic degrees of freedom are rather propagated together with the nuclear ones, which avoids the cost of solving at each step the QM electronic problem. CPMD is thus computationally much more efficient that BOMD. This comes, however, at a cost, as CPMD can produce different results from BOMD. ${ }^{32,34,35}$

The efficiency of BOMD can be majorly improved by building a better initial guess for the SCF equations using information that is available along the trajectory. ${ }^{34,36-38}$

Over the years, many strategies have been proposed to accelerate the SCF procedure in $a b$ initio dynamics. ${ }^{34,36-38}$ Among them, the time-reversible BOMD developed by Niklasson and co-workers ${ }^{39}$ represents an efficient and accurate method, preserving the time-reversal symmetry, thus avoiding systematic errors in energy and gradients and, consequently, memory effects in the nuclear trajectory and an unphysical systematic drift in the system's total energy.

They observed, however, that the perfect time reversibility leads to instabilities under noisy conditions, requiring a sufficiently accurate electron density for longer simulations. To address this issue, they proposed an extended Lagrangian approach, ${ }^{40}$ including the coupling to a fictitious external "dissipative reservoir" to remove the numerical error fluctuations without introducing any significant energy drift or modification of the nuclear forces. In ref 40 the authors show how the dissipative scheme is capable of efficiently removing numerical noise artificially generated by introducing a perturbation during the dynamics of a model system. The same has been done with the lossless approach, producing a noisy trajectory, where the extra noise never disappears.

In the present contribution we apply the extended Lagrangian formalism in the context of the hybrid QM/ AMOEBA BOMD, showing that stable and accurate dynamics can indeed be performed. To do so, we will first recall the working equations for the QM/AMOEBA implementation in a variational formulation. Then a special focus will be given on the presentation of the analytical derivatives of the QM/ AMOEBA energy and on the comparison of the two predictor-corrector schemes by Niklasson et al. ${ }^{39,40}$ that we applied to the computation of the QM part to reduce the computational cost at every hybrid MD step.

\section{POLARIZABLE QM/MM WITH THE AMOEBA FORCE FIELD}

In this section, we will briefly sum up the coupled QM/ AMOEBA equations for a SCF-based QM method. A full derivation can be found in a previous work of some of us. ${ }^{20}$ Detailed information on the AMOEBA force field and the way it treats the polarization problem can be found in the relevant literature. $^{22,41,42}$

Here, we report the QM/AMOEBA variational energy functional

$$
\begin{aligned}
\mathcal{E}(\mathbf{P}, \boldsymbol{\mu}) & =\mathcal{E}^{\mathrm{QM}}(\mathbf{P})+\mathcal{E}^{\mathrm{MM}}(\boldsymbol{\mu})+\mathcal{E}^{\mathrm{Coup}}(\mathbf{P}, \boldsymbol{\mu}) \\
& =\mathcal{E}^{\mathrm{QM}}(\mathbf{P})+\mathcal{E}^{\mathrm{Env}}(\mathbf{P}, \boldsymbol{\mu})
\end{aligned}
$$

where we introduced an environment term $\mathcal{E}^{\text {Env }}$, which is given by the sum of three contributions:

$$
\mathcal{E}^{\mathrm{Env}}=\mathcal{E}^{\mathrm{FF}}+\mathcal{E}_{\mathrm{QM} / \mathrm{MM}}^{\mathrm{El}}(\mathbf{P})+\mathcal{E}_{\mathrm{QM} / \mathrm{MM}}^{\mathrm{Pol}}(\mathbf{P}, \boldsymbol{\mu})
$$

The first term in eq 2 includes the MM bonded and dispersion-repulsion interactions, which depend on neither the electron density nor the induced dipoles. This term includes also the "van der Waals" interactions between the classical and quantum subsystems, which in our implementation are treated with the AMOEBA force field. The second term is given by the electrostatic interaction between the AMOEBA static multipoles and the QM density

$$
\mathcal{E}_{\mathrm{QM} / \mathrm{MM}}^{\mathrm{El}}(\mathbf{P})=\mathbf{q}^{\dagger} \mathbf{V}^{\mathrm{QM}}(\mathbf{P})-\boldsymbol{\mu}_{\mathrm{s}}^{\dagger} \mathbf{E}^{\mathrm{QM}}(\mathbf{P})-\boldsymbol{\Theta}^{\dagger} \mathbf{G}^{\mathrm{QM}}(\mathbf{P})
$$

where $q_{i}, \vec{\mu}_{\mathrm{s}, i}$, and $\boldsymbol{\Theta}_{i}$ are the fixed charges, dipoles, and quadrupoles, respectively, and the potential, field, and field gradient produced by the QM density at the ith $\mathrm{MM}$ atom are each written as the sum of a nuclear and an electronic contribution:

$$
\begin{aligned}
V^{\mathrm{QM}}\left(\vec{r}_{i} ; \mathbf{P}\right)= & \sum_{k}^{N_{\mathrm{QM}}} \frac{Z_{k}}{\left|\vec{r}_{i}-\vec{R}_{k}\right|}+\sum_{\mu \nu}^{N_{\mathrm{b}}} P_{\mu \nu} V_{\mu \nu}\left(\vec{r}_{i}\right) \\
\vec{E}^{\mathrm{QM}}\left(\vec{r}_{i} ; \mathbf{P}\right)= & \sum_{k}^{N_{\mathrm{QM}}} \frac{Z_{k}\left(\vec{r}_{i}-\vec{R}_{k}\right)}{\left|\vec{r}_{i}-\vec{R}_{k}\right|^{3}}+\sum_{\mu \nu}^{N_{\mathrm{b}}} P_{\mu \nu} \vec{E}_{\mu \nu}\left(\vec{r}_{i}\right) \\
{\left[G^{\mathrm{QM}}\right]^{\alpha \beta}\left(\vec{r}_{i} ; \mathbf{P}\right)=} & \sum_{k}^{N_{\mathrm{QM}}} Z_{k}\left(\frac{3\left(r_{i}^{\alpha}-R_{k}^{\alpha}\right)\left(r_{i}^{\beta}-R_{k}^{\beta}\right)}{\left|\vec{r}_{i}-\vec{R}_{k}\right|^{5}}\right. \\
& \left.-\frac{\delta_{\alpha \beta}}{\left|\vec{r}_{i}-\vec{R}_{k}\right|^{3}}\right)+\sum_{\mu \nu}^{N_{\mathrm{b}}} P_{\mu \nu} G_{\mu \nu}^{\alpha \beta}\left(\vec{r}_{i}\right)
\end{aligned}
$$

In eqs 4-6 the index $k$ runs over the $N_{\mathrm{QM}}$ QM nuclei, and $Z_{k}$ and $\vec{R}_{k}$ denote the charge and position of the $k$ th nucleus, respectively. The electronic contributions are written in terms of the density matrix $P_{\mu \nu}$, where $\mu$ and $\nu$ label atomic orbitals, and the integrals $V_{\mu \nu}, \vec{E}_{\mu \nu}$, and $G_{\mu \nu}^{\alpha \beta}$ read

$$
\begin{aligned}
V_{\mu \nu}\left(\vec{r}_{i}\right)= & -\int_{\mathbb{R}^{3}} \frac{\chi_{\mu}(\vec{r}) \chi_{\nu}(\vec{r})}{\left|\vec{r}_{i}-\vec{r}\right|} \mathrm{d}^{3} r \\
\vec{E}_{\mu \nu}\left(\vec{r}_{i}\right)= & -\int_{\mathbb{R}^{3}} \frac{\chi_{\mu}(\vec{r}) \chi_{\nu}(\vec{r})\left(\vec{r}_{i}-\vec{r}\right)}{\left|\vec{r}_{i}-\vec{r}\right|^{3}} \mathrm{~d}^{3} r \\
G_{\mu \nu}^{\alpha \beta}\left(\vec{r}_{i}\right)= & -\int_{\mathbb{R}^{3}} \chi_{\mu}(\vec{r}) \chi_{\nu}(\vec{r}) \\
& \times\left(\frac{3\left(r_{i}^{\alpha}-r^{\alpha}\right)\left(r_{i}^{\beta}-r^{\beta}\right)}{\left|\vec{r}_{i}-\vec{r}\right|^{5}}-\frac{\delta_{\alpha \beta}}{\left|\vec{r}_{i}-\vec{r}\right|^{3}}\right) \mathrm{d}^{3} r
\end{aligned}
$$

Finally, the last term in eq 2 is the variational polarization energy 


$$
\begin{aligned}
& \mathcal{E}_{\mathrm{QM} / \mathrm{MM}}^{\mathrm{Pol}} \\
& \quad=\frac{1}{2} \boldsymbol{\mu}_{\mathrm{d}}^{\dagger} \mathbf{T} \boldsymbol{\mu}_{\mathrm{p}}-\frac{1}{2}\left(\boldsymbol{\mu}_{\mathrm{p}}^{\dagger} \mathbf{E}_{\mathrm{d}}+\boldsymbol{\mu}_{\mathrm{d}}^{\dagger} \mathbf{E}_{\mathrm{p}}\right)-\frac{1}{2}\left(\boldsymbol{\mu}_{\mathrm{p}}+\boldsymbol{\mu}_{\mathrm{d}}\right)^{\dagger} \mathbf{E}^{\mathrm{QM}}(\mathbf{P})
\end{aligned}
$$

This term accounts for the mutual polarization of the QM density and the AMOEBA induced dipoles. A more detailed derivation of the variational formulation of the AMOEBA polarization energy can be found in ref 22, whereas details on the AMOEBA induced dipoles $\boldsymbol{\mu}_{\mathrm{p}}$ and $\boldsymbol{\mu}_{\mathrm{d}}$ and the related electric fields are discussed in ref 41.

The use of a variational formalism makes the derivation of the coupled equations straightforward. The QM/AMOEBA Fock matrix is obtained as the gradient of the functional in 1 with respect to the density matrix:

$$
\tilde{\mathbf{F}}=\frac{\partial \mathcal{E}(\mathbf{P}, \boldsymbol{\mu})}{\partial \mathbf{P}}=\mathbf{F}^{\circ}+\mathbf{F}^{\mathrm{Env}}
$$

In eq $11, \mathbf{F}^{\circ}$ is the standard Fock matrix, whereas $\mathbf{F}^{\mathrm{Env}}$ is the contribution due to the embedding:

$$
F_{\mu \nu}^{\mathrm{Env}}=\mathbf{q}^{\dagger} \mathbf{V}_{\mu \nu}-\boldsymbol{\mu}_{\mathrm{s}}^{\dagger} \mathbf{E}_{\mu \nu}-\boldsymbol{\Theta}^{\dagger} \mathbf{G}_{\mu \nu}-\frac{1}{2}\left(\boldsymbol{\mu}_{\mathrm{p}}+\boldsymbol{\mu}_{\mathrm{d}}\right)^{\dagger} \mathbf{E}_{\mu \nu}
$$

The Fock operator in eq 11 can be used to set up a selfconsistent field procedure. Because it depends on the AMOEBA induced dipoles, the AMOEBA polarization equations need to be solved at each SCF iteration. These are obtained by differentiating the energy functional in eq 1 with respect to both sets of dipoles and setting the derivative to zero, which yields

$$
\begin{aligned}
& \mathbf{T} \boldsymbol{\mu}_{\mathrm{p}}=\mathbf{E}_{\mathrm{p}}+\mathbf{E}^{\mathrm{QM}}(\mathbf{P}) \\
& \mathbf{T} \boldsymbol{\mu}_{\mathrm{d}}=\mathbf{E}_{\mathrm{d}}+\mathbf{E}^{\mathrm{QM}}(\mathbf{P})
\end{aligned}
$$

The two linear systems in eq 13 can be easily solved by using an iterative method, as discussed in detail in refs 42 and 43 . In particular, in the present work we use Jacobi iterations together with the direct inversion in the iterative subspace (DIIS) method $^{44}$ to accelerate convergence. To reduce the drift issue (see discussion in the Numerical Tests section), all simulations are performed with a tight convergence threshold $\left(10^{-8} \mathrm{D}\right)$ for the dipoles.

2.1. Analytical Derivatives of the QM/AMOEBA Energy. We will now proceed with the derivation of the $\mathrm{QM} / \mathrm{AMOEBA}$ gradients. We will focus the discussion on the $\mathrm{QM} / \mathrm{MM}$ interaction energy, and in particular on the electrostatic and polarization terms, given in eqs 3 and 10, respectively. Thanks to our variational formulation, we are only concerned with partial derivatives. By differentiating eq 3 with respect to the coordinates of a $\mathrm{QM}$ nucleus $k$, we get

$$
\frac{\partial \mathcal{E}_{\mathrm{QM} / \mathrm{MM}}^{\mathrm{El}}}{\partial R_{k}^{\alpha}}=\mathbf{q}^{\dagger} \frac{\partial \mathbf{V}^{\mathrm{QM}}}{\partial R_{k}^{\alpha}}-\boldsymbol{\mu}_{\mathrm{s}}^{\dagger} \frac{\partial \mathbf{E}^{\mathrm{QM}}}{\partial R_{k}^{\alpha}}-\boldsymbol{\Theta}^{\dagger} \frac{\partial \mathbf{G}^{\mathrm{QM}}}{\partial R_{k}^{\alpha}}
$$

Equation 14 contains the derivatives of the potential, field and field gradient, as given in eqs 4-6:

$$
\begin{aligned}
& \frac{\partial V^{\mathrm{QM}}\left(\vec{r}_{\vec{r}} ; \mathbf{P}\right)}{\partial R_{k}^{\alpha}}=\frac{Z_{k}\left(r_{i}^{\alpha}-R_{k}^{\alpha}\right)}{\vec{r}_{i}-\vec{R}_{k} k^{3}}+\sum_{\mu \nu}^{N_{\mathrm{b}}} P_{\mu \nu} \frac{\partial V_{\mu \nu}\left(\vec{r}_{i}\right)}{\partial R_{k}^{\alpha}} \\
& \frac{\partial\left[E^{\mathrm{QM}}\right]^{\beta}\left(\vec{r}_{;} ; \mathbf{P}\right)}{\partial R_{k}^{\alpha}}=Z_{k}\left(\frac{3\left(r_{i}^{\alpha}-R_{k}^{\alpha}\right)\left(r_{i}^{\beta}-R_{k}^{\beta}\right)}{\left|\vec{r}_{i}-\vec{R}_{k}\right|^{5}}-\frac{\delta_{\alpha \beta}}{\left|\vec{r}_{i}-\vec{R}_{k}\right|^{\beta}}\right)+\sum_{\mu \nu}^{N_{\mathrm{b}}} P_{\mu \nu} \frac{\partial E_{\mu}^{\beta}(\vec{r})}{\partial R_{k}^{\alpha}} \\
& \frac{\partial\left[G^{\mathrm{QM}}\right] \beta^{\beta \gamma}\left(\vec{r}_{i} ; \mathbf{P}\right)}{\partial R_{k}^{\alpha}}=3 Z_{k} \frac{\left(r_{i}^{\alpha}-R_{k}^{\alpha}\right) \delta_{\beta \gamma}+\left(r_{i}^{\beta}-R_{k}^{\beta}\right) \delta_{\alpha \gamma}+\left(r_{i}^{\gamma}-R_{k}^{\gamma}\right) \delta_{\alpha \beta}}{\left.\right|_{\vec{r}_{i}}-\vec{R}_{k} k^{\beta}} \\
& -15 Z_{k} \frac{\left(r_{i}^{\alpha}-R_{k}^{\alpha}\right)\left(r_{i}^{\beta}-R_{k}^{\beta}\right)\left(r_{i}^{\gamma}-R_{k}^{\gamma}\right)}{\left|\vec{r}_{i}-\vec{R}_{k}\right|^{\gamma}}+\sum_{\mu \nu}^{N_{\mathrm{b}}} P_{\mu \nu} \frac{\partial G_{\mu}^{\beta \gamma}(\vec{r})}{\partial R_{k}^{\alpha}}
\end{aligned}
$$

where the integral derivatives are defined as follows:

$$
\begin{aligned}
\frac{\partial V_{\mu \nu}\left(\vec{r}_{i}\right)}{\partial R_{k}^{\alpha}}= & -\int_{\mathbb{R}^{3}} \frac{\partial\left(\chi_{\mu}(\vec{r}) \chi_{\nu}(\vec{r})\right)}{\partial R_{k}^{\alpha}} \frac{1}{\left|\vec{r}_{i}-\vec{r}\right|} \mathrm{d}^{3} r \\
\frac{\partial E_{\mu \nu}^{\beta}\left(\vec{r}_{i}\right)}{\partial R_{k}^{\alpha}}= & -\int_{\mathbb{R}^{3}} \frac{\partial\left(\chi_{\mu}(\vec{r}) \chi_{\nu}(\vec{r})\right)}{\partial R_{k}^{\alpha}} \frac{\left(r_{i}^{\beta}-r^{\beta}\right)}{\left|\vec{r}_{i}-\vec{r}\right|^{3}} \mathrm{~d}^{3} r \\
\frac{\partial G_{\mu \nu}^{\beta \gamma}\left(\vec{r}_{i}\right)}{\partial R_{k}^{\alpha}}= & -\int_{\mathbb{R}^{3}} \frac{\partial\left(\chi_{\mu}(\vec{r}) \chi_{\nu}(\vec{r})\right)}{\partial R_{k}^{\alpha}}\left(\frac{3\left(r_{i}^{\beta}-r^{\beta}\right)\left(r_{i}^{\gamma}-r^{\gamma}\right)}{\left|\vec{r}_{i}-\vec{r}\right|^{5}}\right. \\
& \left.-\frac{\delta_{\beta \gamma}}{\left|\vec{r}_{i}-\vec{r}\right|^{3}}\right) \mathrm{d}^{3} r
\end{aligned}
$$

Similarly, the gradient of the QM/AMOEBA polarization energy with respect to the position of a QM nucleus is

$$
\frac{\partial \mathcal{E}_{\mathrm{QM} / \mathrm{MM}}^{\mathrm{Pol}}}{\partial R_{k}^{\alpha}}=-\frac{1}{2}\left(\boldsymbol{\mu}_{\mathrm{d}}+\boldsymbol{\mu}_{\mathrm{p}}\right) \frac{\partial \mathbf{E}^{\mathrm{QM}}(\mathbf{P})}{\partial R_{k}^{\alpha}}
$$

with the derivative of the $\mathrm{QM}$ field given in eq 15 .

We now proceed to differentiate the QM/AMOEBA energy with respect to the positions of a MM atom. The electrostatic term gives rise to two different contributions. The first arises from the derivatives of the potential, field, and field gradient, which are, respectively, the field, field gradient, and field second derivative. The second contribution, which we will denote with $\vec{F}_{\text {rot, }, i}$ comes from the matrices that are used to rotate the static dipoles and quadrupoles from the molecular frame to the lab frame. The latter contribution is straightforward, but very cumbersome, and will not be discussed here. The reader can find a complete derivation in ref 42 . The derivative of the electrostatic energy with respect to the position of a classical atoms thus reads

$$
\begin{aligned}
& \frac{\partial \mathcal{E}_{\mathrm{QM} / \mathrm{MM}}^{\mathrm{El}}}{\partial r_{i}^{\alpha}} \\
& \quad=\mathbf{q}^{\dagger}\left[\mathbf{E}^{\mathrm{QM}}\right]^{\alpha}-\left[\mathbf{G}^{\mathrm{QM}}\right]^{\alpha \beta} \boldsymbol{\mu}_{\mathrm{s}}^{\beta}-\left[\mathbf{O}^{\mathrm{QM}}\right]^{\alpha \beta \gamma} \boldsymbol{\Theta}^{\beta \gamma}+F_{\mathrm{rot}, \mathrm{i}}^{\alpha}
\end{aligned}
$$

where we introduced the second field derivative $\mathrm{O}^{\mathrm{QM}}$, whose components are given by

$$
\begin{aligned}
& {\left[O^{\mathrm{QM}}\right]^{\alpha \beta \gamma}\left(\vec{r}_{i} ; \mathbf{P}\right)} \\
& =-\sum_{k}^{N_{\mathrm{QM}}} Z_{k}\left(3 \frac{\left(r_{i}^{\alpha}-R_{k}^{\alpha}\right) \delta_{\beta \gamma}+\left(r_{i}^{\beta}-R_{k}^{\beta}\right) \delta_{\alpha \gamma}+\left(r_{i}^{\gamma}-R_{k}^{\gamma}\right) \delta_{\alpha \beta}}{\left|\vec{r}_{i}-\vec{R}_{k}\right|^{{ }^{\prime}}}\right. \\
& \left.\quad-15 \frac{\left(r_{i}^{\alpha}-R_{k}^{\alpha}\right)\left(r_{i}^{\beta}-R_{k}^{\beta}\right)\left(r_{i}^{\gamma}-R_{k}^{\gamma}\right)}{\left|\vec{r}_{i}-\vec{R}_{k}\right|^{\gamma}}\right)+\sum_{\mu \nu}^{N_{\mathrm{b}}} P_{\mu \nu} O_{\mu \nu}^{\alpha \beta \gamma}\left(\vec{r}_{i}\right)
\end{aligned}
$$

and 


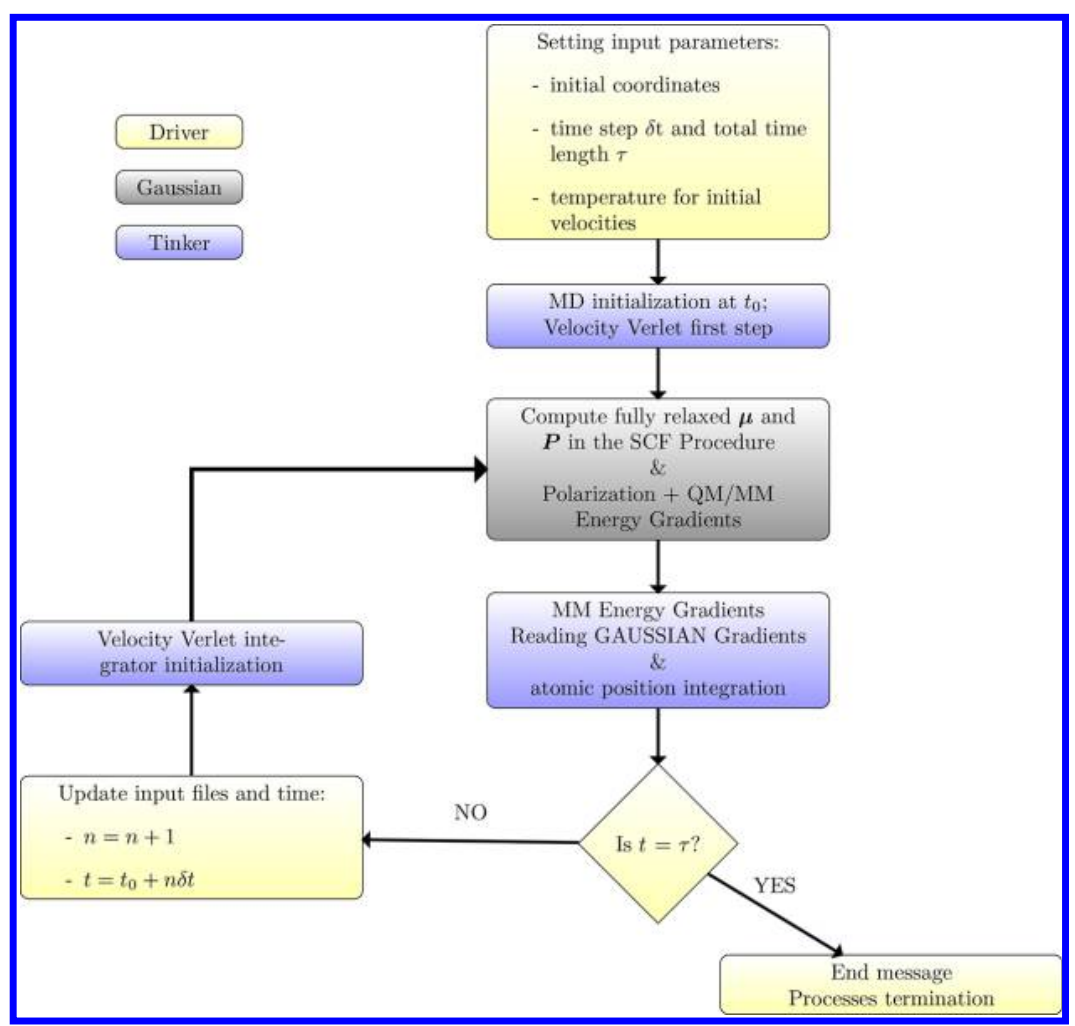

Figure 1. Work-flow diagram of the implementation and Gaussian/TINKER interface. The driver is a bash script overseeing the writing of input files and the exchange of informations between TINKER and Gaussian codes. TINKER is the main program as it collects the energy gradients computed by Gaussian and takes care of the nuclear classical dynamics. Gaussian solves the time-independent Schrödinger equation, computing the electronic PES on-the-fly and its derivative at each nuclear configuration.

$$
\begin{aligned}
& O_{\mu \nu}^{\alpha \beta \gamma}\left(\overrightarrow{r_{i}}\right) \\
& =\int_{\mathbb{R}^{3}} \chi_{\mu}(\vec{r}) \chi_{\nu}(\vec{r})\left(3 \frac{\left(r_{i}^{\alpha}-r^{\alpha}\right) \delta_{\beta \gamma}+\left(r_{i}^{\beta}-r^{\beta}\right) \delta_{\alpha \gamma}+\left(r_{i}^{\gamma}-r^{\gamma}\right) \delta_{\alpha \beta}}{\mid \vec{r}_{i}-\vec{r}^{5}}\right. \\
& \left.\quad-15 \frac{\left(r_{i}^{\alpha}-r^{\alpha}\right)\left(r_{i}^{\beta}-r^{\beta}\right)\left(r_{i}^{\gamma}-r^{\gamma}\right)}{\left|\vec{r}_{i}-\vec{r}\right|^{\prime}}\right) \mathrm{d}^{3} r
\end{aligned}
$$

The derivatives of the QM/AMOEBA polarization energy with respect to the position of a MM atom are given by

$$
\begin{aligned}
\frac{\partial \mathcal{E}_{\mathrm{QM} / \mathrm{MM}}^{\mathrm{Pol}}}{\partial r_{i}^{\alpha}}= & \frac{1}{2} \boldsymbol{\mu}_{\mathrm{d}}^{\dagger} \frac{\partial \mathbf{T}}{\partial r_{i}^{\alpha}} \boldsymbol{\mu}_{\mathrm{p}}-\frac{1}{2}\left(\boldsymbol{\mu}_{\mathrm{p}}^{\dagger} \frac{\partial \mathbf{E}_{\mathrm{d}}}{\partial r_{i}^{\alpha}}+\boldsymbol{\mu}_{\mathrm{d}}^{\dagger} \frac{\partial \mathbf{E}_{\mathrm{p}}}{\partial r_{i}^{\alpha}}\right) \\
& -\frac{1}{2}\left[\mathbf{G}^{\mathrm{QM}}\right]^{\alpha \beta}\left(\boldsymbol{\mu}_{\mathrm{d}}^{\beta}+\boldsymbol{\mu}_{\mathrm{p}}^{\beta}\right)
\end{aligned}
$$

The derivatives of the $\mathbf{T}$ matrix and of the $\mathbf{E}_{\mathrm{d}}$ and $\mathbf{E}_{\mathrm{p}}$ fields are again detailed in ref 42 .

2.2. Acceleration of the QM Part through an Extended Lagrangian Formalism. The so-called extended $\mathrm{BO}$ (XBO) Lagrangian, $\mathcal{L}^{\mathrm{XBO}}$, defined in eq 22 , includes auxiliary electronic degrees of freedom (EDF), here expressed in terms of orthogonal electron density matrices, $\mathbf{P}^{*}$ and its time derivative $\dot{\mathbf{P}}^{*}$, evolving on a harmonic potential centered at the SCF ground-state solution $\mathbf{P}^{\mathrm{SCF}}$. In our implementation, the latter is defined as the QM/AMOEBA SCF ground-state potential $\mathcal{E}\left(\mathbf{R} ; \mathbf{P}^{\mathrm{SCF}} \boldsymbol{\mu}\right)$ for the real EDF. The third and fourth terms of the RHS of eq 22 are the fictitious kinetic and potential energies of the auxiliary EDFs, relative to the fictitious electronic mass $m$ and frequency $\omega$.

$$
\begin{aligned}
\mathcal{L}^{\mathrm{XBO}}\left(\mathbf{R}, \dot{\mathbf{R}}, \mathbf{P}^{*}, \dot{\mathbf{P}}^{*}\right)= & \frac{1}{2} \sum_{I}^{N_{Z}} M_{I} \dot{R}_{I}^{2}-\mathcal{E}\left(\mathbf{R} ; \mathbf{P}^{\mathrm{SCF}}, \boldsymbol{\mu}\right) \\
& +\frac{m}{2} \operatorname{Tr}\left[\dot{\mathbf{P}}^{* 2}\right]-\frac{m \omega}{2} \operatorname{Tr}\left[\left(\mathbf{P}^{\mathrm{SCF}}-\mathbf{P}^{*}\right)^{2}\right]
\end{aligned}
$$

In the limit $m \rightarrow 0, \mathcal{L}^{\mathrm{XBO}} \rightarrow \mathcal{L}^{\mathrm{BO}}$ and the evolution in time of the system is described by the Euler-Lagrange equations of motion

$$
\begin{aligned}
& M_{\mathrm{I}} \ddot{R}_{\mathrm{I}}=-\frac{\partial \mathcal{E}\left(\mathbf{R} ; \mathbf{P}^{\mathrm{SCF}}, \boldsymbol{\mu}\right)}{\partial R_{\mathrm{I}}} \\
& \ddot{\mathbf{P}} *=\omega^{2}\left(\mathbf{P}^{\mathrm{SCF}}-\mathbf{P}^{*}\right)
\end{aligned}
$$

As shown in eq 23, the nuclear degrees of freedom remain unaffected by the extended ones as in a regular BOMD.

The auxiliary EDFs are propagated in a Verlet scheme, as well as the nuclear coordinates, introducing a dissipative force term in a Langevin-like approach, ${ }^{40}$ resulting in the following expression:

$$
\mathbf{P}_{t_{n+1}}^{*}=2 \mathbf{P}_{t_{n}}^{*}-\mathbf{P}_{t_{n-1}}^{*}+k\left(\mathbf{P}_{t_{n}}-\mathbf{P}_{t_{n}}^{*}\right)+\alpha \sum_{l=0}^{K} c_{l} \mathbf{P}_{t_{n-l}}^{*}
$$

where $\alpha$ is the coupling coefficient between the auxiliary EDFs and the external dissipative bath. Further details can be found in ref 40 where also various optimized values for $k, \alpha$, and the linear combination parameters $c_{l}$ are reported. This expression represents the improved density, which will be used as a guess in the SCF procedure. Because the Gaussian code works with nonorthogonal density matrices, we propagate $\tilde{\mathbf{P}}_{t_{n}}^{*}=\mathbf{S}^{1 / 2} \mathbf{P}_{t_{n}}^{*} \mathbf{S}^{1 / 2}$ 
rather than $\mathbf{P}_{t_{n}}^{*}$, as proposed by Skylaris et al. ${ }^{45}$ The initial guess is again given by eq 25 , after multiplying $\tilde{\mathbf{P}}_{t_{n+1}}^{*}$ by $\mathbf{S}^{-1 / 2}$ on the left and right.

\section{NUMERICAL TESTS}

To perform hybrid polarizable QM/MM MD simulation, an interface between a locally modified version of Gaussian ${ }^{27}$ and Tinker (and Tinker-HP) has been created. The work flow is described in details in Figure 1. In our implementation, Gaussian is used to solve the $\mathrm{QM} / \mathrm{MM}$ equations and to compute the electrostatic and polarization QM/MM energy and forces. This allows us to minimize communication between Gaussian and Tinker and will allow us to exploit our previous FMM-based linear scaling implementation of the polarizable electrostatics. ${ }^{19}$ A fully linear scaling implementation is currently under active investigation.

Tinker/Tinker-HP computes all MM nonelectrostatic terms and, given the total forces, integrates the equations of motions. The QM/MM driver handles the communication between Gaussian and Tinker and works directly on the input/output of each program.

As a test case we perform a series of QM/AMOEBA MD simulations using free (nonperiodic) boundary conditions of the alanine dipeptide (ADP) in a cubic box of 631 waters $(1912$ atoms overall, Figure 2). The dipeptide represents the QM subsystem in our hybrid approach and is treated at the B3LYP/ 6-31G level, whereas the water molecules are treated at the AMOEBA level.

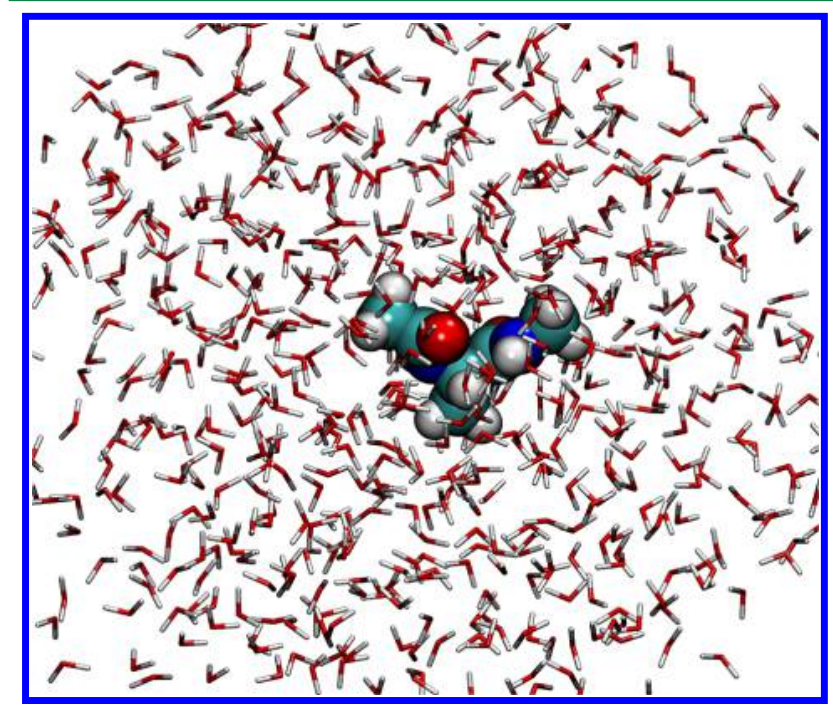

Figure 2. Snapshot extracted from an MD run. The ADP molecule in the center is the QM subsystem, surrounded by water molecules described with the AMOEBA polarizable FF.

We ran several MD trajectories in the NVE ensemble, using a time step $\delta t=0.5$ fs for a total simulation time of $1 \mathrm{ps}$, without imposing any boundary condition. In general, this can lead to various issues, and in particular, to the evaporation of the system, but because our tests were performed for very short simulation times, comparable to that of a low-energy vibration, we are not concerned here with these kinds of problems. For longer simulations, a harmonic repulsive constraint ${ }^{46}$ is available to avoid evaporation artifacts. Indeed, the watersolute cluster can be confined by a spherical boundary with a van der Waals soft wall represented by a 12-6 Lennard-Jones potential, which can be set to a fixed buffer distance of $2.5 \AA$ outside the specified radius.

Here we discuss the tests we carried out to analyze the effect that the environment, the extrapolation scheme and the SCF convergence threshold have on the energy conservation. The SCF convergence thresholds tested are $10^{-3}, 10^{-5}$, and $10^{-8}$. Note that convergence in Gaussian is based on the root-meansquare (RMS) variation of the electron density, rather than the energy. The loosest value $\left(10^{-3}\right)$ is chosen to mimic a strongly noised dynamics, with poorly converged density and inaccurate gradients. Because our simulations are run in the microcanonical ensemble, we cannot directly control temperature, except for the sampling of the initial velocities, obtained from a Maxwell-Boltzmann distribution.

The two extrapolation schemes employed are that of Niklasson et al. ${ }^{39}$ (henceforth labeled TR-BOMD) and that of eq 25 (XL-BOMD) with $K=7$. Although different values of $K$ have been used in the literature, ${ }^{45,47}$ we follow the work of Niklasson and co-workers, ${ }^{48}$ where tight-binding DFT is applied to different amino acids and different $K$ values are compared. Finally, we test the effect of the environment by comparing the simulations in water with gas-phase simulations, keeping all the other settings unchanged.

We compare the drift in total energy and the noise in each of the 12 resulting trajectories. The drift is assumed to be linear in time and is computed as the slope of the best line interpolating the time sequence of the energy values. The noise is evaluated as the root-mean square of the energy fluctuations after removing the drift. ${ }^{38} \mathrm{We}$ also report the pseudotemperature of the EDFs, ${ }^{45,49}$ computed as $\operatorname{Tr}\left[\dot{\mathbf{X}}^{2}\right] / N_{\mathrm{e}}$, where $\mathbf{X}=\mathbf{P} *$ or $\mathbf{P}^{\mathrm{SCF}}$, the dot indicates time derivatives (performed numerically), and $N_{\mathrm{e}}$ is the number of electrons. As starting geometry, we use a snapshot extracted from an Amber classical MD and set a low temperature $(50 \mathrm{~K})$ for the initial velocity sampling. Because the temperature determines the amount of energy per vibrational degree of freedom, the energy oscillation amplitudes are temperature dependent. High-temperature simulations have the effect of increasing the noise, thus affecting the accuracy of our estimation of the energy drift, as shown in the Supporting Information where the results of a $300 \mathrm{~K}$ dynamics is reported (Figure S1).

The results are reported in Table 1. In Figure 3, we plot the total energy as $E_{\text {tot }}(t)-E_{\text {avg }}$ for each simulation. Panels $A$ and $\mathrm{B}$ refer to gas-phase and QM/AMOEBA simulations. The red lines represent the energy of TR-BO, using the $10^{-3}$ SCF convergence threshold. The insets clearly show that the energy diverges, reaching $\sim 2000 \mathrm{mE} E_{\mathrm{H}}$ after $315 \mathrm{fs}$ in the gas phase and $355 \mathrm{fs}$ in water. Due to the high noise (92220 and $74810 \mu E_{\mathrm{H}}$, respectively) and inaccuracy in the drift determination, the results are not meaningful and conclusion cannot be drawn on the dynamics conservativity.

Using the XL-BO extrapolation (Figure 3, blue line, panels A, $\mathrm{B})$, the results are more stable, as the energy does not diverge in either the gas or condensed phase, although the drift is quite large ( 2000 and $\sim 1000 \mu E_{\mathrm{H}} / \mathrm{ps}$ ). Furthermore, the average number of SCF cycles along the whole trajectory, $\bar{N}$, in XL-BO is almost half that in TR-BO ( $\sim 3$ for XL-BO vs $\sim 5$ for TR$\mathrm{BO})$, as expected. Because the dissipative extended Lagrangian approach removes the numerical noise, the dynamics is more stable, even though the noise remains rather large $(\sim 3$ and $\sim 4$ $\mathrm{m} E_{\mathrm{H}}$ in the gas phase and QM/AMOEBA, respectively).

The different behavior of the two approaches can be easily understood by plotting the pseudotemperature of the auxiliary 
Table 1. Comparison of the Average Number of SCF Cycles $(\bar{N})$, Total Energy Drift, and Noise between Different Convergence Criteria, for Both Gas-Phase and QM/AMOEBA BOMD, Using Eq 7 in Ref 39 [(TR-BOMD) and Eq 25 (XL-BOMD) with $K=$ 7 for the SCF Guess Extrapolation]

\begin{tabular}{|c|c|c|c|c|c|c|c|}
\hline \multirow[b]{2}{*}{$\operatorname{conv}\left(10^{-N}\right)$} & \multirow[b]{2}{*}{ method } & \multicolumn{3}{|c|}{ gas phase } & \multicolumn{3}{|c|}{ QM/AMOEBA } \\
\hline & & $\bar{N}$ & $\operatorname{drift}\left(\mu E_{\mathrm{H}} / \mathrm{ps}\right)$ & noise $\left(\mu E_{\mathrm{H}}\right)$ & $\bar{N}$ & $\operatorname{drift}\left(\mu E_{\mathrm{H}} / \mathrm{ps}\right)$ & noise $\left(\mu E_{\mathrm{H}}\right)$ \\
\hline \multirow[t]{2}{*}{3} & $\mathrm{TR}^{-\mathrm{BO}^{a}}$ & 5.4 & $72340 \pm 79320$ & 92220 & 5.1 & $54930 \pm 53770$ & 74810 \\
\hline & $\mathrm{XL}-\mathrm{BO}$ & 2.8 & $2090 \pm 470$ & 3190 & 2.7 & $1060 \pm 530$ & 3630 \\
\hline \multirow[t]{2}{*}{5} & TR-BO & 5.18 & $-9.0 \pm 0.67$ & 4.6 & 5.46 & $-82 \pm 3.2$ & 22 \\
\hline & XL-BO & 3.62 & $-0.66 \pm 0.74$ & 5.0 & 3.78 & $-72 \pm 3.2$ & 22 \\
\hline \multirow[t]{2}{*}{8} & TR-BO & 7.29 & $-0.20 \pm 0.19$ & 1.3 & 7.40 & $-70 \pm 3.2$ & 22 \\
\hline & XL-BO & 7.83 & $-0.21 \pm 0.19$ & 1.3 & 8.09 & $-70 \pm 3.2$ & 22 \\
\hline
\end{tabular}

${ }^{a}$ In this case the trajectory explodes around $315 \mathrm{fs}$ in the gas phase and $355 \mathrm{fs}$ in water, so the average number of SCF cycles, the drift, and the noise are computed over these time intervals.

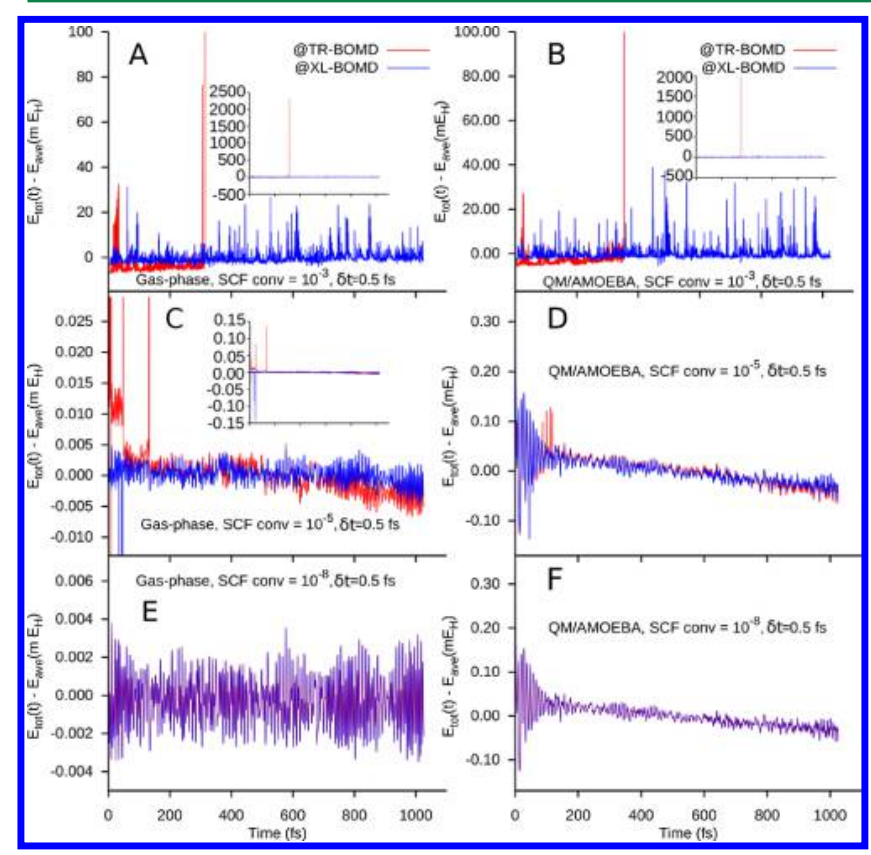

Figure 3. Energy variation $\left(\mathrm{m} E_{\mathrm{H}}\right)$ along $1 \mathrm{ps}$ long trajectories. The plots on the left (A, C, E) refer to gas-phase simulations; those on the right $(\mathrm{B}, \mathrm{D}, \mathrm{F})$, to simulations in water solution. Convergence thresholds are $10^{-3}$ (plots $\mathrm{A}$ and $\mathrm{B}$ ), $10^{-5}$ (C and $\mathrm{D}$ ), and $10^{-8}$ (E and $\mathrm{F})$. The insets in plots $\mathrm{A}, \mathrm{B}$, and $\mathrm{C}$ show the full energy fluctuation range. and real EDFs in the two cases. Figure 4 shows the overheating of the auxiliary variables for TR-BO, which diverge significantly and quite rapidly (after $\sim 200 \mathrm{fs}$ ) from the real converged density. This leads to a loss of computational efficiency, because the SCF procedure is no longer able to reach convergence quickly. In the XL-BO case the two pseudotemperatures remain quite close to each other. However, even if the XL-BO extrapolation stabilizes the trajectory, the still large values of the energy drift and noise prevent one from considering it as safe and reliable at such low convergence thresholds.

Increasing the convergence threshold to $10^{-5}$, we obtain consistently better results, and we can observe a non-negligible difference between TR- and XL-BO (Table 1 and Figure 3, panels $\mathrm{C}$ and $\mathrm{D}$ ). In the gas phase (red lines) both approaches give substantially no drift; nevertheless, XL-BO still exhibits a better behavior, showing a smaller noise value and $\sim 30 \%$ less SCF cycles on average.

Comparing these results with the QM/AMOEBA ones (blue lines), we find that the drift and the noise are 2 and 1 orders of magnitude greater (XL-BO and TR-BO, respectively) than the corresponding values in the gas phase. This is mainly due to the effect of the MM polarization, because the iterative resolution for the induced dipoles causes the term $\frac{\partial E}{\partial \mu}$ to be not exactly zero, with a consequent nonzero Hellmann-Feynman residual force. TR- and XL-BO schemes are almost equivalent in the condensed phase, where the energy drift in our 1 ps test (panel $\mathrm{D}$ in Figure 3$)$ is noticeable although very small $\left(10^{-1} \mathrm{~m} E_{\mathrm{H}}\right)$.

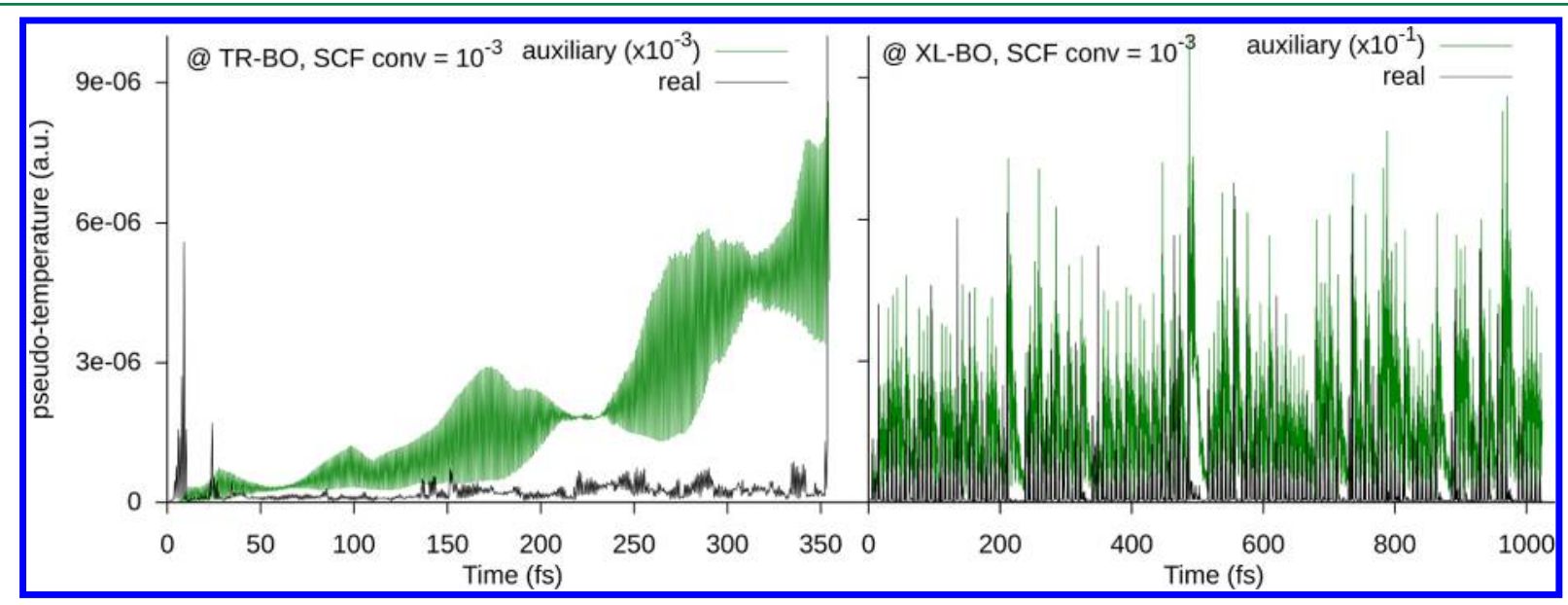

Figure 4. Comparison between the QM/AMOEBA auxiliary and real EDF pseudotemperature for SCF convergence set to $10^{-3}$ in arbitrary units (au). The values for the auxiliary EDFs are scaled by a factor $10^{-3}$ for TR-BO and $10^{-1}$ for XL-BO to obtain comparable scales. 
The XL-BO still shows a 30\% gain in SCF efficiency, which can be explained again with the EDFs pseudotemperature analysis (see Supporting Information, Figure S2). We should also observe that, compared to the case of a regular BOMD, the save in elapsed time is almost $50 \%$.

Moving to the tightest convergence criterion $\left(10^{-8}\right)$, almost no difference can be found by comparing TR- and XL-BO within the same environment, and also no differences with XL$\mathrm{BO}$ when the SCF convergence is set to $10^{-5}$. The same happens for the pseudotemperature of the electronic variables, which we are not reporting. As a final remark, we note that, given the small size of the QM system we employed for our tests, the impact of the different convergence thresholds on the overall computational cost of a simulation is limited. However, we expect that being able to use a less conservative threshold will speed up real life applications of a factor approximately proportional to the reduction in SCF cycles.

We can then conclude that

- for XL-BO, convergence for energy and energy gradients is reached already with a much lower convergence criterion than that usually suggested when QM forces have to be computed $\left(10^{-8}\right)$;

- as expected from theory, the perfectly lossless TRBOMD is affected by the noise due to numerical approximations in the SCF solution. Nevertheless, as soon as well-converged densities are used, it is efficient in reducing the number of SCF cycles and produces a very stable simulation.

\section{CONCLUSIONS}

In this contribution, we presented an implementation of the analytical gradients of the polarizable QM/AMOEBA energy and of the machinery needed to perform efficient and stable molecular dynamics simulations. In particular, we used density functional theory as a quantum mechanical method, and the extended Born-Oppenheimer Lagrangian technique to provide an improved guess to the self-consistent field solver. Such a technique allows one to achieve energy-conserving and stable simulations, also offering remarkable computational advantages. We tested it by running several MD simulations on a small system, the alanine dipedptide (QM) solvated by water molecules (AMOEBA), comparing the TR- and XL-BO schemes using different SCF thresholds. We found that the $\mathrm{XL}-\mathrm{BO}$ is the most effective approach when a moderately accurate convergence threshold is used, thanks to its capability to avoid the "heating" of the auxiliary EDFs, which remain close to the real ones along the dynamics. Our implementation is presently limited to systems where the $\mathrm{MM}$ portion is not covalently bonded to the QM one and is therefore suited to study solute/solvent systems. A further implementation allowing us to treat covalently bonded QM and MM systems is currently under investigation, aiming to apply the link atom strategy as well as a pseudopotential approach, following the implementation of ref 23. This work represents a first step toward large scale polarizable $\mathrm{QM} / \mathrm{MM} \mathrm{MD}$ simulations and reactivity studies. More efficient and parallel computational strategies need to be used to extend the applicability of the method. In particular, to be able to treat large to very large systems, comprising up to tens of thousands of atoms in the classical subsystem, a linear-scaling, parallel, and efficient implementation of AMOEBA will be required, such as the one available in the Tinker HP suite of programs, ${ }^{19,43,50,51}$ developed by some of us. It should be noted that polarizable $\mathrm{QM} / \mathrm{MM}$ simulations are in principle more expensive than their nonpolarizable counterparts. However, the QM part of the calculation usually dominates the overall simulation cost, making the increased cost associated with the classical part not an issue. Another aspect that needs to be addressed is the handling of boundary conditions. For nonperiodic, polarizable $\mathrm{QM} / \mathrm{MM} \mathrm{MD}$ simulations, the use of a polarizable continuum as a boundary is particularly attractive. ${ }^{52,53}$ Purely classical polarizable MD simulations within a polarizable continuum have already been discussed by some of $\mathrm{us}^{22}$ and are made possible by ddCOSMO, a fast, domain-decomposition-based implementation $^{54,55}$ of the conductor-like screening model. ${ }^{56}$ An efficient and scalable implementation of a three-layer QM/ AMOEBA/ddCOSMO model for molecular dynamics simulation is currently being investigated.

\section{ASSOCIATED CONTENT}

\section{S Supporting Information}

The Supporting Information is available free of charge on the ACS Publications website at DOI: 10.1021/acs.jctc.7b00572.

Figure $\mathrm{S} 1$ and $\mathrm{S} 2$, reporting the pseudotemperature comparison between TR- and XL-BO for SCF convergence $10^{-5}$ and for $50 \mathrm{~V}$ s $300 \mathrm{~K}$ dynamics, respectively $(\mathrm{PDF})$

\section{AUTHOR INFORMATION}

\section{Corresponding Authors}

*F. Lipparini. E-mail: filippo.lipparini@unipi.it.

*B. Mennucci. E-mail: benedetta.mennucci@unipi.it.

*J.-P. Piquemal. E-mail: jpp@lct.jussieu.fr.

\section{ORCID}

Daniele Loco: 0000-0002-6808-7515

Filippo Lipparini: 0000-0002-4947-3912

Benedetta Mennucci: 0000-0002-4394-0129

Jean-Philip Piquemal: 0000-0001-6615-9426

\section{Present Address}

${ }^{\text {II } D i p a r t i m e n t o ~ d i ~ C h i m i c a ~ e ~ C h i m i c a ~ I n d u s t r i a l e, ~ U n i v e r s i t a ̀ ~ d i ~}$ Pisa, via G. Moruzzi 13, I-56124 Pisa, Italy.

Notes

The authors declare no competing financial interest.

\section{ACKNOWLEDGMENTS}

This work was supported in part by French funds managed by CalSimLab and the ANR within the Investissements $d$ Avenir program under reference ANR-11-IDEX-0004-02. J.-P.P. and L.L. are grateful for support by the Direction Generale de 1 Armement (DGA) Maitrise NRBC of the French Ministry of Defense. The authors are indebted to Étienne Polack for providing the updated QM/MM driver. D.L. is grateful to Chris-Kriton Skylaris for helpful discussions on the extrapolation of the density matrix. J.-P.P. thanks Matt Challacombe and Christophe Raynaud for fruitful discussions

\section{REFERENCES}

(1) Gresh, N.; Cisneros, G. A.; Darden, T. A.; Piquemal, J.-P. Anisotropic, Polarizable Molecular Mechanics Studies of Inter- and Intramolecular Interactions and Ligand-Macromolecule Complexes. A Bottom-Up Strategy. I. Chem. Theorv Comput. 2007, 3, 1960-1986.

(2) Ponder, J. W.; Wu, C.; Ren, P.; Pande, V. S.; Chodera, J. D.; Schnieders, M. J.; Haque, I.; Mobley, D. L.; Lambrecht, D. S.; DiStasio, 
R. A.; Head-Gordon, M.; Clark, G. N. I.; Johnson, M. E.; HeadGordon, T. Current Status of the AMOEBA Polarizable Force Field. L. Phvs. Chem. B 2010, 114, 2549-2564.

(3) Mancini, G.; Brancato, G.; Barone, V. Combining the Fluctuating Charge Method, Non-periodic Boundary Conditions and Metadynamics: Aqua Ions as Case Studies. J. Chem. Theory Comput. 2014, 10, 1150-1163.

(4) Bryce, R. A.; Buesnel, R.; Hillier, I. H.; Burton, N. A. A solvation model using a hybrid quantum mechanical/molecular mechanical potential with fluctuating solvent charges. Chem. Phys. Lett. 1997, 279, 367-371.

(5) Lipparini, F.; Barone, V. Polarizable Force Fields and Polarizable Continuum Model: A Fluctuating Charges/PCM Approach. 1. Theory and Implementation. I. Chem. Theorv Comput. 2011, 7, 3711-3724.

(6) Lipparini, F.; Cappelli, C.; Barone, V. Linear response theory and electronic transition energies for a fully polarizable QM/Classical Hamiltonian. I. Chem. Theory Comput. 2012, 8, 4153-4165.

(7) Lipparini, F.; Cappelli, C.; Scalmani, G.; De Mitri, N.; Barone, V. Analytical First and Second Derivatives for a Fully Polarizable QM/ Classical Hamiltonian. I. Chem. Theory Comput. 2012, 8, 4270-4278. (8) Lipparini, F.; Cappelli, C.; Barone, V. A gauge invariant multiscale approach to magnetic spectroscopies in condensed phase: General three-layer model, computational implementation and pilot applications. I. Chem. Phvs. 2013, 138, 234108.

(9) Lamoureux, G.; MacKerell, A. D. J.; Roux, B. A simple polarizable model of water based on classical Drude oscillators. J. Chem. Phys. 2003, 119, 5185-5197.

(10) Boulanger, E.; Thiel, W. Solvent Boundary Potentials for Hybrid QM/MM Computations Using Classical Drude Oscillators: A Fully Polarizable Model. I. Chem. Theory Comput. 2012, 8, 4527-4538.

(11) Thompson, M. A.; Schenter, G. K. Excited States of the Bacteriochlorophyll b Dimer of Rhodopseudomonas viridis: A QM/ MM Study of the Photosynthetic Reaction Center That Includes MM Polarization. I. Phvs. Chem. 1995, 99, 6374-6386.

(12) Jensen, L.; van Duijnen, P. T.; Snijders, J. G. A discrete solvent reaction field model for calculating molecular linear response properties in solution. I. Chem. Phvs. 2003, 119, 3800.

(13) Nielsen, C. B.; Christiansen, O.; Mikkelsen, K. V.; Kongsted, J. Density functional self-consistent quantum mechanics/molecular mechanics theory for linear and nonlinear molecular properties: Applications to solvated water and formaldehyde. J. Chem. Phys. 2007, 126,154112 .

(14) Illingworth, C. J. R.; Parkes, K. E. B.; Snell, C. R.; Ferenczy, G. G.; Reynolds, C. A. Toward a Consistent Treatment of Polarization in Model QM/MM Calculations. I. Phvs. Chem. A 2008, 112, 1215112156.

(15) Curutchet, C.; Muñoz-Losa, A.; Monti, S.; Kongsted, J.; Scholes, G. D.; Mennucci, B. Electronic Energy Transfer in Condensed Phase Studied by a Polarizable QM/MM Model. J. Chem. Theory Comput. 2009, 5, 1838-1848.

(16) Si, D.; Li, H. Analytic energy gradient in combined timedependent density functional theory and polarizable force field calculation. I. Chem. Phvs. 2010, 133, 144112.

(17) Steindal, A. H.; Ruud, K.; Frediani, L.; Aidas, K.; Kongsted, J. Excitation Energies in Solution: The Fully Polarizable QM/MM/PCM Method. J. Phvs. Chem. B 2011, 115, 3027-3037.

(18) Zeng, Q.; Liang, W. Analytic energy gradient of excited electronic state within TDDFT/MMpol framework: Benchmark tests and parallel implementation. I. Chem. Phys. 2015, 143, 134104.

(19) Caprasecca, S.; Jurinovich, S.; Lagardère, L.; Stamm, B.; Lipparini, F. Achieving Linear Scaling in Computational Cost for a Fully Polarizable MM/Continuum Embedding. J. Chem. Theory Comput. 2015, 11, 694-704.

(20) Loco, D.; Polack, É; Caprasecca, S.; Lagardère, L.; Lipparini, F.; Piquemal, J.-P.; Mennucci, B. A QM/MM Approach Using the AMOEBA Polarizable Embedding: From Ground State Energies to Electronic Excitations. I. Chem. Theory Comput. 2016, 12, 3654-3661.
(21) Lipparini, F.; Scalmani, G.; Mennucci, B.; Cancès, E.; Caricato, M.; Frisch, M. J. A variational formulation of the polarizable continuum model. I. Chem. Phvs. 2010, 133, 014106.

(22) Lipparini, F.; Lagardère, L.; Raynaud, C.; Stamm, B.; Cancès, E.; Mennucci, B.; Schnieders, M.; Ren, P.; Maday, Y.; Piquemal, J. P. Polarizable molecular dynamics in a polarizable continuum solvent. $J$. Chem. Theorv Comput. 2015, 11, 623-634.

(23) Kratz, E. G.; Walker, A. R.; Lagardère, L.; Lipparini, F.; Piquemal, J.-P.; Andrés Cisneros, G. LICHEM: A QM/MM program for simulations with multipolar and polarizable force fields. J. Comput. Chem. 2016, 37, 1019-1029.

(24) Dziedzic, J.; Mao, Y.; Shao, Y.; Ponder, J.; Head-Gordon, T.; Head-Gordon, M.; Skylaris, C.-K. TINKTEP: A fully self-consistent, mutually polarizable QM/MM approach based on the AMOEBA force field. I. Chem. Phvs. 2016, 145, 124106.

(25) Mao, Y.; Shao, Y.; Dziedzic, J.; Skylaris, C.-K.; Head-Gordon, T.; Head-Gordon, M. Performance of the AMOEBA Water Model in the Vicinity of QM Solutes: A Diagnosis Using Energy Decomposition Analysis. I. Chem. Theory Comput. 2017, 13, 1963-1979.

(26) Ponder, J. W. TINKER, Software Tools for Molecular Design. http://dasher.wustl.edu/tinker.

(27) Frisch, M. J.; Trucks, G. W.; Schlegel, H. B.; Scuseria, G. E.; Robb, M. A.; Cheeseman, J. R.; Scalmani, G.; Barone, V.; Mennucci, B.; Petersson, G. A.; Nakatsuji, H.; Caricato, M.; Li, X.; Hratchian, H. P.; Izmaylov, A. F.; Bloino, J.; Janesko, B. G.; Lipparini, F.; Zheng, G.; Sonnenberg, J. L.; Liang, W.; Hada, M.; Ehara, M.; Toyota, K.; Fukuda, R.; Hasegawa, J.; Ishida, M.; Nakajima, T.; Honda, Y.; Kitao, O.; Nakai, H.; Vreven, T.; Montgomery, J. A., Jr.; Peralta, J. E.; Ogliaro, F.; Bearpark, M.; Heyd, J. J.; Brothers, E.; Kudin, K. N.; Staroverov, T.; Keith, T.; Kobayashi, R.; Normand, J.; Raghavachari, K.; Rendell, A.; Burant, J. C.; Iyengar, S. S.; Tomasi, J.; Cossi, M.; Rega, N.; Millam, J. M.; Klene, M.; Knox, J. E.; Cross, J. B.; Bakken, V.; Adamo, C.; Jaramillo, J.; Gomperts, R.; Stratmann, R. E.; Yazyev, O.; Austin, A. J.; Cammi, R.; Pomelli, C.; Ochterski, J. W.; Martin, R. L.; Morokuma, K.; Zakrzewski, V. G.; Voth, G. A.; Salvador, P.; Dannenberg, J. J.; Dapprich, S.; Parandekar, P. V.; Mayhall, N. J.; Daniels, A. D.; Farkas, O.; Foresman, J. B.; Ortiz, J. V.; Cioslowski, J.; Fox, D. J. Gaussian Development Version, Revision H.36; Gaussian Inc.: Wallingford, CT, 2010.

(28) Brunk, E.; Rothlisberger, U. Mixed Quantum Mechanical/ Molecular Mechanical Molecular Dynamics Simulations of Biological Systems in Ground and Electronically Excited States. Chem. Rev. 2015, 115, 6217-6263.

(29) Jensen, M. Ø.; Röthlisberger, U.; Rovira, C. Hydroxide and Proton Migration in Aquaporins. Biophvs. I. 2005, 89, 1744-1759.

(30) Alfonso-Prieto, M.; Biarnés, X.; Vidossich, P.; Rovira, C. The Molecular Mechanism of the Catalase Reaction. J. Am. Chem. Soc. 2009, 131, 11751-11761.

(31) Boero, M.; Ikeda, T.; Ito, E.; Terakura, K. Hsc70 ATPase: An Insight into Water Dissociation and Joint Catalytic Role of $\mathrm{K}+$ and Mg2+ Metal Cations in the Hydrolysis Reaction. J. Am. Chem. Soc. 2006, 128, 16798-16807.

(32) Marx, D.; Hutter, J. Modern Methods and Algorithms of Quantum Chemistry, 2nd ed.; John von Neumann Institute for Computing: Jülich, Germany, 2000.

(33) Car, R.; Parrinello, M. Unified Approach for Molecular Dynamics and Density-Functional Theory. Phys. Rev. Lett. 1985, 55, 2471-2474.

(34) Payne, M. C.; Teter, M. P.; Allan, D. C.; Arias, T. A.; Joannopoulos, J. D. Rev. Mod. Phvs. 1992, 64, 1045-1097.

(35) Tangney, P. On the theory underlying the Car-Parrinello method and the role of the fictitious mass parameter. J. Chem. Phys. 2006, 124, 044111.

(36) Millam, J.; Bakken, V.; Chen, W.; Hase, L.; Schlegel, H. B. J. Chem. Phys. 1999, 111, 3800-3805.

(37) Pulay, P.; Fogarasi, G. Chem. Phys. Lett. 2004, 386, 272-278.

(38) Herbert, J.; Head-Gordon, M. Phys. Chem. Chem. Phys. 2005, 7, 3269-3275. 
(39) Niklasson, A. M. N.; Tymczak, C. J.; Challacombe, M. TimeReversible Born-Oppenheimer Molecular Dynamics. Phys. Rev. Lett. 2006, 97, 123001.

(40) Niklasson, A. M. N.; Steneteg, P.; Odell, A.; Bock, N.; Challacombe, M.; Tymczak, C. J.; Holmström, E.; Zheng, G.; Weber, V. Extended Lagrangian Born-Oppenheimer molecular dynamics with dissipation. I. Chem. Phvs. 2009, 130, 214109.

(41) Ponder, J. W.; Wu, C.; Ren, P.; Pande, V. S.; Chodera, J. D.; Schnieders, M. J.; Haque, I.; Mobley, D. L.; Lambrecht, D. S.; DiStasio, R. A., Jr; Head-Gordon, M.; Clark, G. N. I.; Johnson, M. E.; HeadGordon, T. Current Status of the AMOEBA Polarizable Force Field. J. Phvs. Chem. B 2010, 114, 2549-2564.

(42) Lipparini, F.; Lagardére, L.; Stamm, B.; Cancés, E.; Schnieders, M.; Ren, P.; Maday, Y.; Piquemal, J.-P. Scalable Evaluation of Polarization Energy and Associated Forces in Polarizable Molecular Dynamics: I. Toward Massively Parallel Direct Space Computations. J. Chem. Theorv Comput. 2014, 10, 1638-1651.

(43) Lagardère, L.; Lipparini, F.; Polack, E.; Stamm, B.; Cancès, E.; Schnieders, M.; Ren, P.; Maday, Y.; Piquemal, J.-P. Scalable Evaluation of Polarization Energy and Associated Forces in Polarizable Molecular Dynamics: II. Toward Massively Parallel Computations Using Smooth Particle Mesh Ewald. I. Chem. Theory Comput. 2015, 11, 2589-2599.

(44) Pulay, P. Convergence acceleration of iterative sequences. the case of scf iteration. Chem. Phys. Lett. 1980, 73, 393-398.

(45) Vitale, V.; Dziedzic, J.; Albaugh, A.; Niklasson, A. M. N.; HeadGordon, T.; Skylaris, C.-K. Performance of extended Lagrangian schemes for molecular dynamics simulations with classical polarizable force fields and density functional theory. J. Chem. Phys. 2017, 146, 124115.

(46) Marjolin, A.; Gourlaouen, C.; Clavaguéra, C.; Ren, P. Y.; Wu, J. C.; Gresh, N.; Dognon, J.-P.; Piquemal, J.-P. Toward accurate solvation dynamics of lanthanides and actinides in water using polarizable force fields: from gas-phase energetics to hydration free energies. Theor. Chem. Acc. 2012, 131, 1198.

(47) Niklasson, A. M. N.; Steneteg, P.; Bock, N. Extended Lagrangian free energy molecular dynamics. I. Chem. Phvs. 2011, 135, 164111.

(48) Zheng, G.; Niklasson, A. M. N.; Karplus, M. Lagrangian formulation with dissipation of Born-Oppenheimer molecular dynamics using the density-functional tight-binding method. J. Chem. Phys. 2011, 135, 044122.

(49) Albaugh, A.; Demerdash, O.; Head-Gordon, T. An efficient and stable hybrid extended Lagrangian/self-consistent field scheme for solving classical mutual induction. I. Chem. Phvs. 2015, 143, 174104.

(50) Narth, C.; Lagardère, L.; Polack, E.; Gresh, N.; Wang, Q.; Bell, D. R; Rackers, J. A.; W, P. J.; Ren, P.; Piquemal, J.-P. Scalable improvement of SPME multipolar electrostatics in anisotropic polarizable molecular mechanics using a general short-range penetration correction up to quadrupoles. J. Comput. Chem. 2016, 37, 494-505.

(51) Aviat, F.; Levitt, A.; Stamm, B.; Maday, Y.; Ren, P.; Ponder, J. W.; Lagardére, L.; Piquemal, J.-P. Truncated Conjugate Gradient: An Optimal Strategy for the Analytical Evaluation of the Many-Body Polarization Energy and Forces in Molecular Simulations. J. Chem. Theory Comput. 2017, 13, 180-190.

(52) Brancato, G.; Rega, N.; Barone, V. Reliable molecular simulations of solute-solvent systems with a minimum number of solvent shells. I. Chem. Phvs. 2006, 124, 214505.

(53) Brancato, G.; Nola, A. D.; Barone, V.; Amadei, A. A mean field approach for molecular simulations of fluid systems. J. Chem. Phys. 2005, 122, 154109.

(54) Cancès, E.; Maday, Y.; Stamm, B. Domain Decomposition for Implicit Solvation Models. J. Chem. Phys. 2013, 139, 05411.

(55) Lipparini, F.; Stamm, B.; Cancès, E.; Maday, Y.; Mennucci, B. Fast Domain Decomposition Algorithm for Continuum Solvation Models: Energy and First Derivatives. J. Chem. Theory Comput. 2013, 9, 3637-3648.

(56) Klamt, A.; Schuurmann, G. COSMO: a new approach to dielectric screening in solvents with explicit expressions for the screening energy and its gradient. J. Chem. Soc., Perkin Trans. 2 1993, 799-805. 